To be published in: Journal of Economic Behavior \& Organization

\title{
Man's search for meaning: The case of Legos
}

\author{
Dan Ariely ${ }^{\mathbf{a}}$ \\ Emir Kamenica $\mathbf{b}$, \\ Dražen Prelec ${ }^{a}$
}

${ }^{a}$ MIT Sloan School of Management, 38 Memorial Drive, Cambridge, MA 02142, United States

$\mathrm{b}$ University of Chicago Graduate School of Business, 5807 South Woodlawn Avenue, Chicago, IL 60637, United States

*Corresponding author. Tel.: +1 7738348690 .

E-mail addresses: ariely@mit.edu (D. Ariely),emir.kamenica@ChicagoGSB.edu (E. Kamenica),dprelec@mit.edu (D.Prelec). 


\begin{abstract}
We investigate how perceived meaning influences labor supply. In a laboratory setting, we manipulate the perceived meaning of simple, repetitive tasks and find a strong influence on subjects' labor supply. Despite the fact that the wage and the task are identical across the conditions in each experiment, subjects in the less meaningful conditions exhibit reservation wages that are consistently much higher than the subjects in the more meaningful conditions. The result replicates across different types of tasks. Moreover, in the more meaningful conditions, subjects' productivity influences labor supply more strongly.
\end{abstract}


If human nature felt no...satisfaction (profit apart) in constructing a factory, a railway, a mine or a farm, there might not be much investment as a result of cold calculation...Enterprise only pretends to itself to be mainly motivated by the statement in its own prospectus.

- John Maynard Keynes

Most children think of their potential future occupations in terms of what they will be (firemen, doctors, etc.), not merely what they will $d o$ for a living. Many adults also think of their job as an integral part of their identity. At least in the United States, "What do you do?" has become as common a component of an introduction as the anachronistic "How do you do?" once was, yet identity, pride, and meaning are all left out from standard models of labor supply. This omission is understandable: identity, pride, and meaning are difficult to quantify and are thus hard to incorporate into the empirically driven field of labor economics.

In this article, we focus on minimal perceived meaning by the labor producing force and investigate how it influences labor supply in controlled laboratory experiments. Our intention is to compare situations with no meaning (or as low a level of meaning as we can create) with situations having some small additional meaning. Thus, our investigation will focus not on occupations highly endowed with meaning, like medicine or teaching, but on the least-commondenominator of meaningfulness that is shared by virtually all compensated activities.

With this goal in mind, our conceptualization of meaning is intentionally basic; we view labor as meaningful to the extent that (a) it is recognized and/or (b) has some point or purpose. Recognition means that some other person acknowledges the completion of the work. Such recognition does not have to be linked to any financial incentives or to any non-tangible rewards such as praise or appreciation. Purpose means that the employees understand how their work might be linked, even tangentially, to some objectives. This does not mean that the workers 
necessarily endorse or care about these objectives, but only that they can relate their labor to a more general objective. We propose that these twin factors are two of the hidden motivational foundations of meaning-in-labor.

To study the impact of minimal meaning on labor supply in a laboratory setting, we take simple repetitive tasks and, in some conditions, drain them as much as we can of all possible meaning. Although the wages and the physical task requirements are kept the same across conditions, we consistently find that subjects in the "pointless" conditions reveal higher reservation wages (i.e., demand more payment for same work). We also find that perceived meaning enhances the relationship between labor supply and skill. Specifically, in the more meaningful condition, subjects' productivity (as measured by the speed with which they accomplish the task) has a stronger effect on labor supply.

Previous literature on the impact of meaning on behavior is quite sparse, especially within economics. Frankl (2006 [1962], p. 99) in his book Man's Search for Meaning argues that meaning is the "primary motivational force in man."1 Loewenstein's (1999) study of mountaineering literature similarly examines the role of meaning as an incentive. ${ }^{2}$ Less directly, our results are complementary to the literature on compensating differentials, which has attempted to measure the impact of amenities on wages. ${ }^{3}$ Preston (1989) and Leete (2001) look at whether individuals accept lower wages to work in the non-profit sector, while Stern (1999) examines whether scientists are willing to take a wage cut in order to be able to publish their work. By using an experimental design, we avoid the problem of possible correlation between

1 The subtitle of our paper is an unintended play on words: Frankl's therapeutic doctrine is called logo therapy, after the Greek word logos, which denotes meaning.

${ }^{2}$ We owe the initial quotation of Keynes to George Loewenstein.

${ }^{3}$ A useful, though out-of-date, survey of this literature is provided by Rosen (1987). 
amenities and unmeasured worker ability, which is an issue in the cross-sectional studies. More importantly, our results suggest that the standard conception of an amenity is much too narrow and concrete. In our experiments the tasks are equally meaningful (or perhaps we should say equally futile) across the conditions by any objective accounting of the task requirements, yet by placing a thin veil over the futility of the task, we are able to induce a greater willingness to work. Meaning is cheap, so to speak, but ignoring the dimension of meaning may be quite expensive, for employer and for society.

\section{Experiment 1: the impact of meaning on labor supply}

\section{Experimental design}

Our first study looks at whether the supply of labor for a tedious and repetitive task can be modulated by superficial manipulations of meaning. The subjects were MIT students who responded to announcements about the experiment that were posted in the student center, where the experiment also took place. Each subject participated in the experiment alone, without the presence of other subjects. Subjects were randomly assigned to one of three conditions: Acknowledged $(\mathrm{N}=35)$, Ignored $(\mathrm{N}=35)$, or Shredded $(\mathrm{N}=34)$. Subjects were unaware of the other conditions. The basic task was the same in all three conditions: subjects were initially given a sheet of paper with a seemingly random sequence of letters and told that they would be paid \$0.55 for finding 10 instances of two consecutive letters 's. ${ }^{, 4}$ Having completed the first page, they were then asked whether they would be willing to complete a second page for $\$ 0.50(5 \varnothing$ less). The process continued, with wages declining by $5 \varnothing$ per sheet, until the subject decided to stop working. This ended the experimental session. Subjects then received payment for all sheets.

\footnotetext{
${ }^{4}$ For each sheet, we generated a random sequence of letters and then modified the sequence manually to ensure there are exactly 10 instances of two consecutive letters 's.'
} 
Since we paid the subjects on a per-unit rather than per-hour basis, we accordingly measure labor supply in terms of units produced, not hours worked. The instructions for Experiment 1 are provided in Appendix A of the Supplementary material.

In the Acknowledged condition, the subjects were asked to write their name on each sheet prior to starting the task. The instructions explained that after completing the task, they would hand the sheet over to the experimenter who would examine it and file it away in a folder.

In the Ignored condition, the subjects were not instructed to write their name on the sheets, and in fact none did so. Moreover, the instructions explained that, after the subject completed the task, the experimenter would place the sheet on a high stack of papers. The experimenter in fact did so without examining the completed sheets.

The Shredded condition was the same as the Ignored condition except that the instructions explained that the completed sheets would be immediately put through a paper shredder. As the subjects turned in the sheets, the experimenter shredded them without a glance.

The subjects could cheat in all the conditions, given the absence of monitoring. Moreover, the incentives to cheat are arguably higher in the Ignored condition and even higher in the Shredded condition where the lack of monitoring was particularly salient. ${ }^{5}$ Moreover, in the Shredded condition, cheating was not only impossible to detect, but is obviously of no consequence since the sheets were immediately destroyed. To the extent that economic theory makes any directional predictions here, it would seem to predict the highest reservation wage in the Acknowledged condition, which requires more conscientious attention to a dull task, and lowest in the Shredded condition, where cheating is both possible and apparently inconsequential.

\footnotetext{
${ }^{5}$ We examined the sheets afterwards: there was no cheating in either the Acknowledged or the Ignored condition. The destruction of the sheets prevents us from determining whether there was cheating in the Shredded condition.
} 
Results

The results were exactly opposite of these predictions: the subjects exhibited the lowest average reservation wage in the Acknowledged condition (14.85ф), a higher one in the Ignored condition $(26.14 \phi)$, and the highest in the Shredded condition $(28.29 \phi)$. In other words, in the three conditions the subjects completed an average of 9.03, 6.77, and 6.34 sheets and received an average total of $\$ 3.01, \$ 2.60$, and $\$ 2.42$. Fig. 1 shows the histograms of the number of sheets completed in each condition. As the histograms show, almost half of the subjects in the Acknowledged condition were willing to work until the wage dropped all the way to zero.

The Wilcoxon rank-order test reveals that labor supply was significantly greater in the Acknowledged than in the Ignored condition (exact one-sided $p$-value $<0.001$ ), while the difference between the Ignored and Shredded conditions is not statistically significant (exact one-sided $p$-value $=0.24)$. The magnitude of the difference between the Acknowledged and the other two conditions is quite striking: the subjects exhibit a reservation wage that is almost twice as large when their work is not acknowledged. The difference between Acknowledged and Ignored condition is not nearly as strong, ${ }^{6}$ which is somewhat surprising. The act of shredding the sheets without even looking at them is such blatant, unnatural violence toward the product of subjects' labor that one might expect the subjects to respond much more to it than to the treatment in the Ignored condition, yet the difference between those two conditions is minor while the effect of being acknowledged is strikingly high.

${ }^{6}$ Though the difference between the strength of the effects is only marginally significant (exact one-sided p-value $=0.064$ ). 


\section{Experiment 2: a replication and examination of the role of productivity}

\section{Experimental design}

In our second experiment, we replicate the result with a physical task of a different nature and additionally examine how meaning affects the relationship between productivity and labor supply.

The subjects were male undergraduates at Harvard University, recruited via posters around the university. ${ }^{7}$ Each subject participated in the experiment alone, without the presence of other subjects. Subjects were randomly assigned to one of the two conditions, Meaningful $(\mathrm{N}=$ 20) and Sisyphus $(\mathrm{N}=20)$, and were unaware of the other condition. The procedure was similar to that used in Experiment 1. In each of the two conditions, subjects received payments for assembling Bionicle Lego models according to a declining unit wage schedule. Each Bionicle consisted of 40 separate pieces, with written instructions on how to assemble them into a figure. There was only one way to combine the pieces, and no subject had trouble following the assembly instructions. The mean time to build the first Bionicle was around $10 \mathrm{~min}$. Before deciding whether to build each Bionicle, the subjects were told how much they had earned up to that point and how much they would earn for making another Bionicle. The subjects were paid $\$ 2.00$ for the first Bionicle, $\$ 1.89$ (11ф less) for the second one, and so on linearly. For the 20th, as well as for any subsequent Bionicles, they received $\$ 0.02$. The only decision the subjects made was when to stop making Bionicles. At that point, they were paid and the experimental session was over. During the experiment, we measured how long it took each subject to build each Bionicle. The instructions are provided in Appendix B of the Supplementary material. In the Meaningful condition, after the subject would build each Bionicle, he would place

\footnotetext{
7 We recruited subjects of only one gender to reduce ambient variance.
} 
it on the desk in front of him, and the experimenter would give him a new box with new Bionicle pieces. Hence, as the session progressed, the completed Bionicles would accumulate on the desk. In the Sisyphus condition, there were only two boxes. After the subject completed the first Bionicle and began working on the second, the experimenter would disassemble the first Bionicle into pieces and place the pieces back into the box. Hence, the Bionicles could not accumulate; after the second Bionicle, the subject was always rebuilding previously assembled pieces that had been taken apart by the experimenter. This was the only difference between the two conditions. ${ }^{8}$ Furthermore, all the Bionicles were identical, so the Meaningful condition did not provide more variety than the Sisyphus one.

Results

Despite the fact that the physical task requirements and the wage schedule were identical in the two conditions, the subjects in the Meaningful condition built significantly more Bionicles than those in the Sisyphus condition. In the Meaningful condition, subjects built an average of 10.6 Bionicles and received an average of $\$ 14.40$, while those in the Sisyphus condition built an average of 7.2 Bionicles and earned an average of $\$ 11.52$. The histograms for the number of Bionicles made in each condition are reported in Fig. 2.

The Wilcoxon rank-order test reveals that the reservation wage was significantly greater in the Sisyphus than in the Meaningful condition (exact one-sided $p$-value $=0.005$ ). The median subject in the Sisyphus condition stopped working at $\$ 1.40$, while the median subject in the Meaningful condition stopped at $\$ 1.01$. Hence, the difference is economically as well as statistically significant, as the Sisyphus manipulation increased the median reservation wage by

${ }^{8}$ In order to avoid the situation where the number of Bionicles completed is more salient in the Meaningful condition, the experimenter maintained a visible count in both conditions. 
about 40 percent.

While the magnitude of the difference in the implied reservation wages is somewhat surprising, the existence of the effect conforms with intuition. ${ }^{9}$ However, a priori intuitions about possible differences in the strength of the relationship between willingness to work and productivity are more varied.

If the effect of perceived meaning enters the disutility of labor additively, productivity (speed of building a Bionicle) should have the same effect on labor supply in the two conditions. However, one might argue that the standard model of labor supply, where the agent trades costs of effort and lost time for the benefit of earned wages, applies most directly to a setting where the agent has no emotional relationship with his or her work. Under this view, since high productivity implies a lower time cost, we might expect to find a stronger correlation between labor supply and the speed of assembling Legos in the Sisyphus condition than in the Meaningful condition: the alienated workers in the Sisyphus condition might be more likely to make a cold calculation between their wage and their time cost, making more units if it takes them less time to build each one.

Alternatively, one could argue that the subjects in the Sisyphus condition simply become disenchanted with their work and become insensitive to the tradeoff between time and money. Hence, we might expect to find a stronger correlation in the Meaningful condition. The data unequivocally support the latter view.

The Spearman correlation between the number of Bionicles produced and average speed

\footnotetext{
${ }^{9}$ In a separate experiment, we paid subjects to predict the difference in the number of Bionicles built in the two conditions. The vast majority of subjects predicted the direction of the effect correctly, though the average predicted magnitude was roughly half of the actual one.
} 
of building them is $0.838(\mathrm{p}<0.001)$ in the Meaningful condition and $0.251(\mathrm{p}=0.29)$ in the Sisyphus condition. This difference in the strength of the relationship between productivity and labor supply is highly significant (exact two-sided $p$-value $=0.018$ ). Note, however, that the relationship between productivity and labor supply includes a selection effect: subjects become faster as they build more Bionicles. To address this issue, we also use the speed of building the first Bionicle as the measure of productivity. ${ }^{10} \mathrm{We}$ get qualitatively the same results. Specifically, the correlation is $0.454(\mathrm{p}<0.05)$ in the Meaningful condition and $-0.274(\mathrm{p}=0.24)$ in Sisyphus. The exact two-sided $p$-value for the difference is 0.031 . Hence, even when the selection effect cannot play a role, subjects' productivity influences labor supply more strongly in the Meaningful condition.

\section{Conclusion}

Speculation about the relationship between identity and labor supply goes back to Marx's (1983 [1844]) notion of alienation of labor. For Marx, an alienated laborer is separated from his own activities, from the goals of his labor, and from the process of production. This makes work an external activity that consequently does not allow the laborer to fulfill himself and find identity in his work. The importance of identity in economic decision making was recently emphasized by Akerlof and Kranton (2000), who analyze the role of identity in the formation of social structures more generally.

Occupations that are traditionally regarded as meaningful (medicine, art, science, pedagogy) are invariably associated with large and 'noble' goals. Individuals presumably derive satisfaction from a feeling that their work promotes these goals, which in turn leads to lower reservation wages. In this light, the standard models of labor supply are certainly consistent with

\footnotetext{
10 As expected, this measure of productivity is not significantly different across the two conditions.
} 
the results of our two experiments; if perceived meaning is an amenity, disutility of effort may depend on the perceived meaning of the task, and it may do so non-additively, changing the elasticity as well as the level of labor supply. However, we believe that a focus on specific amenities, relevant only to some professions, can limit our understanding of meaning-in-labor. In our view, meaning, at least in part, derives from the connection between work and some purpose, however insignificant or irrelevant that purpose may be to the worker's personal goals. When that connection is severed, when there is no purpose, work becomes absurd, alienating, or even demeaning.

In fact, although the work in the less meaningful conditions may have seemed pointless, in reality it was not pointless at all. Subjects knew that they were participating in a research experiment and advancing the cause of science, albeit in a small way. The objectives of the experiment were inscrutable, but they were equally inscrutable in the meaningful and meaningless conditions. To the extent that participation in a scientific experiment was an 'amenity,' there is no reason why the size of the amenity should differ across conditions. The critical difference, of course, was that the pointlessness of the task requirement was made much more salient in the meaningless conditions. The background question, "Why am I doing this?", is difficult to evade if an individual is in a situation where one's work is repeatedly undone.

Demanding performance of an activity that is manifestly pointless can be construed as an (otherwise incomprehensible) exercise of power for its own sake. The best way to show 'who is boss' is precisely to order someone to toil for no reason. Anecdotal evidence for this idea is evident in many movies about prison life where the guards force the prisoners to dig holes and fill them back up or to move large rocks from one part of a field to the other and back.

If indeed small effects of meaning in terms of recognition and purpose can have large 
effects on labor in the marketplace, this would bring into question the wisdom of breaking tasks into components and training the labor force to specialize in one such component, since this would reduce the ability of the laborers to understand the consequences of their effort and their ability to perceive the completion of the overall goal. Our results may also have prescriptive implications for educating laborers about the goals of their work. Although such education might take some time from the workers' busy schedule and cost the organization, it might prove beneficial.

The work presented here also sheds some new light on the relationship between monitoring and effort. Many researchers, such as Falk and Kosfeld (2006), have suggested that close supervision of workers might undermine intrinsic motivation. Our Experiment 1 suggests that the way in which monitoring is framed crucially influences its effect on motivation. If perceived as interest in the worker, supervision might improve worker morale rather than induce a feeling of lost autonomy. Thus, monitoring that is accompanied by increased meaning (recognition, education, acknowledgment) might not only eliminate the negative side effects of control, but also increase workers' effort and motivation. 


\section{References}

Akerlof, G.A., Kranton, R.E., 2000. Economics and identity. Quarterly Journal of Economics $115,715-753$.

Falk, A., Kosfeld, M., 2006. The hidden costs of control. American Economic Review 96, 16111630.

Frankl, V.E., 2006. Man’s Search for Meaning. Boston Beacon Press (1962).

Leete, L., 2001. Whither the nonprofit wage differential? Estimates from the 1990 Census. Journal of Labor Economics 19, 136-170.

Loewenstein, G., 1999. Because it is there: the challenge of mountaineering . . for utility theory. Kyklos 52, 315-343.

Marx, K., 1983. Economico-philosophical manuscripts of 1844. In: Kamenka, E. (Ed.), The Portable Karl Marx. Penguin Books, New York (1844).

Preston, A.E., 1989. The nonprofit worker in a for-profit world. Journal of Labor Economics 7 , 438-463.

Rosen, S., 1987. The theory of equalizing differences. In: Ashenfelter, O., Layard, R. (Eds.), Handbook of Labor Economics, vol. 1. Elsevier Science, New York, pp. 641-692.

Stern, S., 1999. Do scientists pay to be scientists? NBER Working Paper No. 7410. 
Figures

Fig. 1. Number of sheets completed in the Acknowledged, Ignored, and Shredded conditions.

(a)

Acknowledged
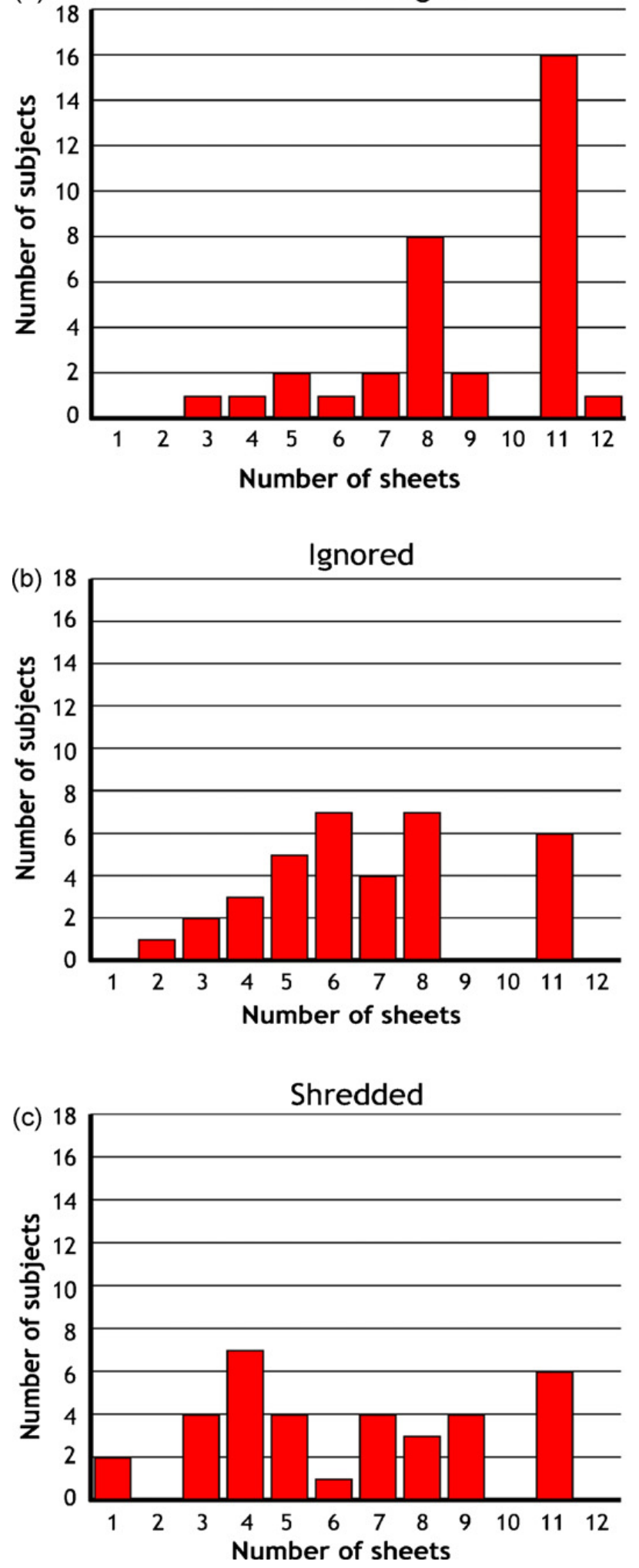
Fig. 2. Number of Bionicles completed in the Meaningful and Sisyphus conditions.
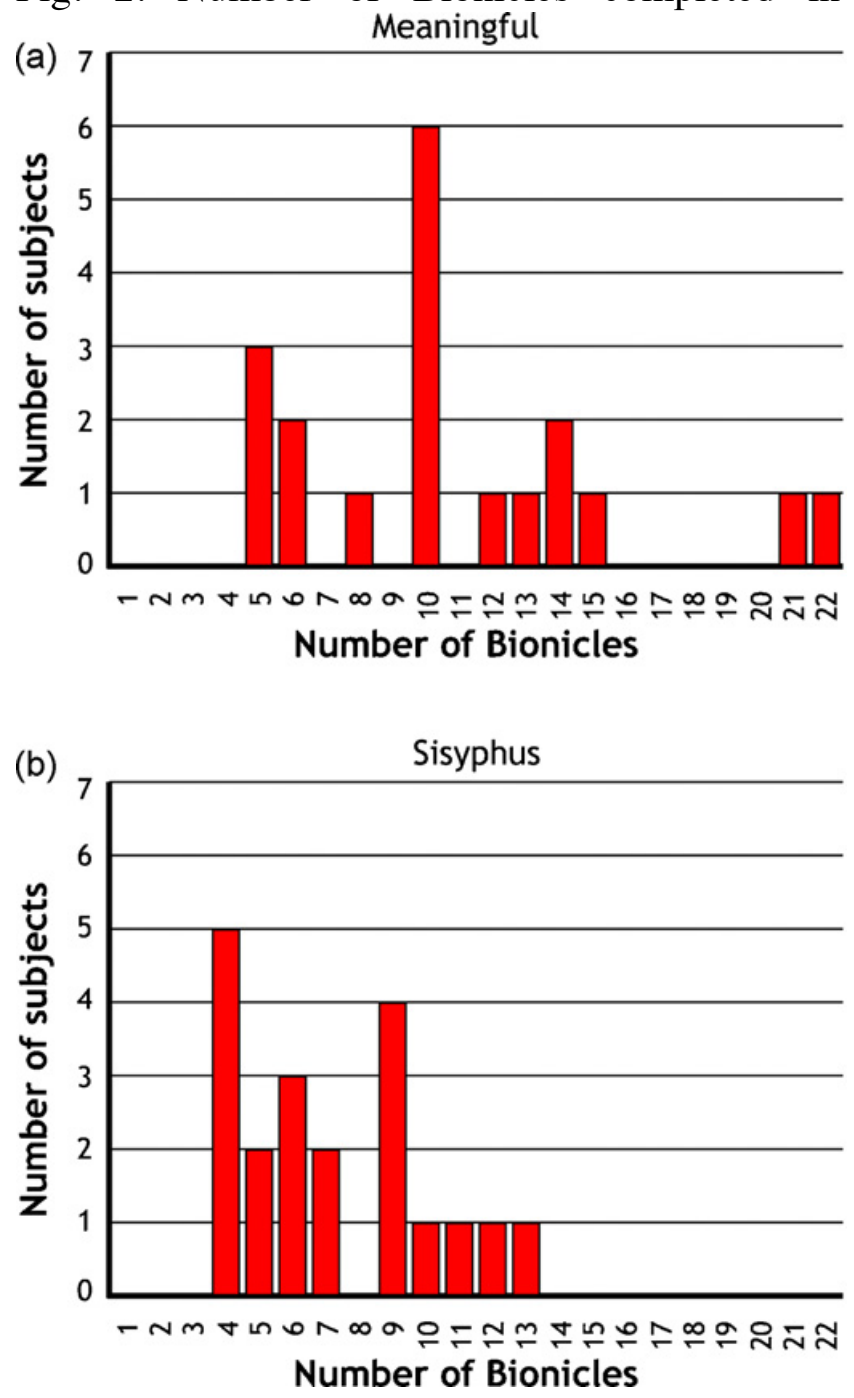
Appendix A: Instructions and sample sheet of letter task used in Experiment 1

In this study you will be given sheets of paper filled with letters (see sample below).

mly yboggunednomcykntsioqedcssnrrrulgssrddjtyeunrjtnnvhg qncgmmoryflqtgwwcukczpmrccxjteflpkkngnazzyimezgeettevt knzvxxmgsswxzifhupigtqsbgvwumkhssylauyymwbll1ilgiioinw phumjknwcaupzzdavaifoodrcrkecjfxfqdhqekdfhhnajqatpks lc ns sgjofhzbwqeizvqqfincmjaiwdiivivvbgnkdnqvphpeehkgxnvsugi qrtsqrjhekbdceazju uxaqvtcdjjizvnobtbnricckpqwpi ipr punfhj by x m mrghna ov vddena e exebagyihgokkpis scxgohqmtyzzkrfm $x$ e h t y v q itgxnkrqanokbdssdqdbf

Each sheet has ten sets of two letters S one next to each other (SS). Completing a sheet means that you find all ten pairs and mark them. You will get $55 \not$ for the first sheet and once you finish it, you will be asked if you want to complete another sheet for $50 \varnothing, 45 \varnothing$ etc (see payment table below). You can continue with this for as long as you wish.

\begin{tabular}{ccc}
\hline Sheet \# & Payment $(\phi)$ & Total $(\$)$ \\
\hline 1 & $55 \varnothing$ & $\$ 0.55$ \\
2 & $50 \varnothing$ & $\$ 1.05$ \\
3 & $45 \varnothing$ & $\$ 1.50$ \\
4 & $40 \varnothing$ & $\$ 1.90$ \\
5 & $35 \varnothing$ & $\$ 2.25$ \\
6 & $30 \varnothing$ & $\$ 2.55$ \\
7 & $25 \varnothing$ & $\$ 2.80$ \\
8 & $20 \varnothing$ & $\$ 3.00$ \\
9 & $15 \varnothing$ & $\$ 3.15$ \\
10 & $10 \varnothing$ & $\$ 3.25$ \\
11 & $5 \varnothing$ & $\$ 3.30$ \\
$12+$ & $0 \varnothing$ & $\$ 3.30$ \\
\hline
\end{tabular}

In the Acknowledged condition subjects were also given the following instructions:

Please write your name at the top of each sheet before you start. After you complete a sheet hand it to the experimenter who will look it over and file it away in a folder.

In the Ignored condition subjects were also given the following instructions:

After you complete each sheet hand it to the experimenter who will place it on top of his stack of papers.

The Shredded condition subjects were also given the following instructions: After you complete each sheet hand it to the experimenter who will immediately put it through the paper shredder. 
Appendix B: Instructions for the Bionicles task used in Experiment 2

Thank you for participating in this study on Bionicle Lego models.

Each box contains 40 pieces that can be assembled into a Bionicle. The pieces should be combined as in the attached instructions to create a stable Bionicle.

Your earnings for the study depend on how many Bionicles you build. You will get the most money for the first Bionicle, less for the second, even less for the third and so on. Before you build each Bionicle the research assistant will tell you how much money you can make by building another Bionicle.

It's up to you how many Bionicles you build. The only rules are:

1) Work on one Bionicle at a time.

2) Don't start a new Bionicle before you finish the one you are working on.

3) When you finish a Bionicle, give it to the research assistant.

When you don't want to make any more Bionicles, let the research assistant know. At that point you will be paid. 